\title{
Morphometric study of fossa ovale in human cadaveric hearts: embryological and clinical relevance
}

\author{
Prajakta Kishve ${ }^{1}$, Rohini Motwani ${ }^{2}$ \\ ${ }^{1}$ Department of Anatomy, ESIC Medical College \& Hospital, Hyderabad, ${ }^{2}$ Department of Anatomy, All India Institute of Medical Sciences, Bibinagar, \\ Hyderabad, India
}

\begin{abstract}
Atrial septal defect (ASD) is the 5th common congenital abnormality at birth. Secundum atrial defect and patent foramen ovale ( $\mathrm{PFO}$ ) are the most common atrial septal defects. In this setting, the anatomical functional characterization of the interatrial septum seems to be of paramount importance not only for device selection but also for therapeutic intervention. This study was carried out to evaluate the morphometric parameters of fossa ovale (FOv) in the human adult cadaveric hearts. For this study, 50 normal cadaveric human hearts available in the department of Anatomy over the period of 3 years were used where size, position, shape, nature of the FOv was noted. The size of the fossa was measured and prominence, location, and extent of the limbus fossa ovalis were observed. The probe patency of foramen ovale (FO) was confirmed. In most specimens, the fossa was oval (80\%), the average transverse diameter was $24.21 \mathrm{~mm}$, and the vertical diameter $26.84 \mathrm{~mm}$. In $84 \%$ rim was raised. In $56 \%$ of cases, the fossa was present at the middle of the interatrial septum. The patency of foramen was observed in $3 \%$. The findings of the present study provide pertinent information on the morphology of the FOv, which may be useful for device selection in treating ASDs and PFO. This would definitely help the clinicians in a deeper understanding of the region as very few cadaveric studies are available in the literature at present.
\end{abstract}

Key words: Atrial septal defect, Cadaver, Foramen ovale, Heart, Patent foramen ovale

Received October 30, 2020; Revised November 19, 2020; Accepted December 10, 2020

\section{Introduction}

\section{Embryology of interatrial septum and fossa ovale}

The interior of the right atrium (RA) consists of three main parts i.e. a smooth posterior part, Sinus venarum, rough anterior part, and interatrial septum [1]. The formation of atrial septa is initiated at a late stage of cardiac development (from day 60 onwards). The septal wall presents

\footnotetext{
Corresponding author:

Rohini Motwani iD

Department of Anatomy, All India Institute of Medical Sciences, Bibinagar, Hyderabad-508126, India
}

E-mail: rohinimotwani@gmail.com an oval depression above and to the left of the orifice of the inferior vena cava (IVC) named as fossa ovale (FOv) (fossa ovalis). Its floor is the primary atrial septum, the septum primum, a sickle-shaped crest that descends from the roof of the primitive atrium, begins to divide the atrium in two but leaves an opening, the ostium primum, for communication between the two sides (Fig. 1) Later, when the ostium primum is obliterated by fusion of the septum primum with the endocardial cushions, the ostium secundum is formed by cell death that creates an opening in the septum primum. This ostium secundum supplies prenatal blood flow from the right to the left atrium (LA) to bypass premature pulmonary circulation. Finally, a septum secundum forms, but an interatrial opening, the oval foramen (or foramen ovale, FO) persists (Fig. 2). When the upper part of the septum primum

\section{Copyright (c) 2021. Anatomy \& Cell Biology}

This is an Open Access article distributed under the terms of the Creative Commons Attribution Non-Commercial License (http://creativecommons.org/licenses/by-nc/4.0/) which permits unrestricted non-commercial use, distribution, and reproduction in any medium, provided the original work is properly cited. 

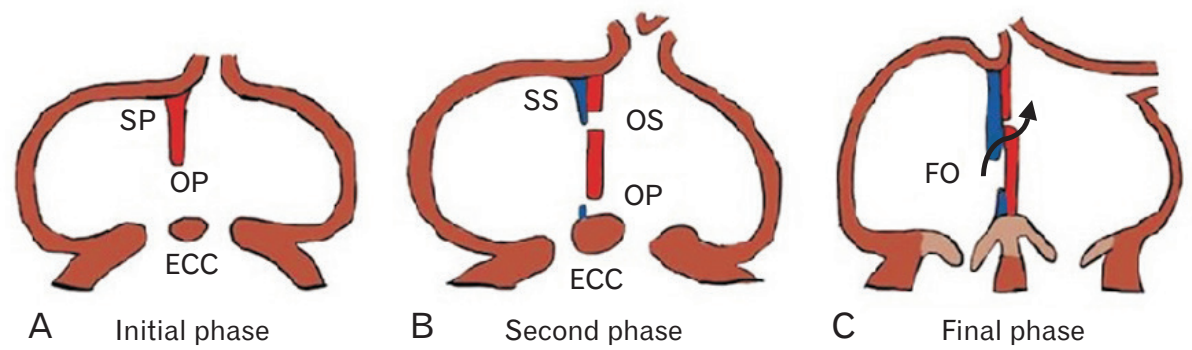

Fig. 1. Normal formation of IAS. (A) SP a sickle-shaped crest that descends from the roof of the primitive atrium. Opening between SP and ECC is OP. (B) SP fusing with ECC, perforation in the upper part of SP forms OS. New fold appears from roof as SS. (C) Opening between lower end of SS and upper end of SP is FO. ECC, endocardial cushion; FO, foramen ovale; IAS, inter atrial septum; OP, ostium primum; OS, ostium secundum; SP, septum primum; SS, septum secundum.

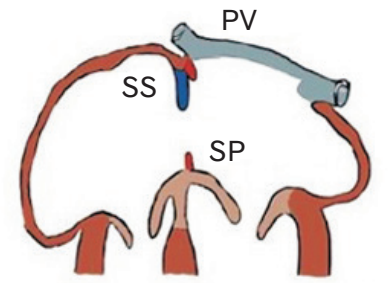

A Excessive resorption of SP

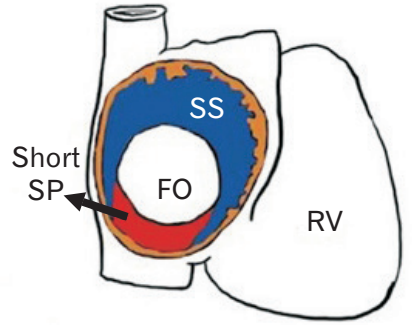

D

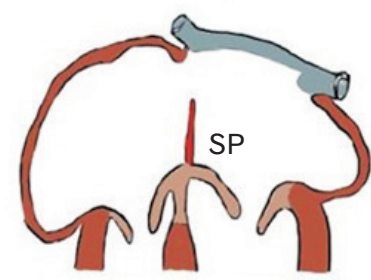

B

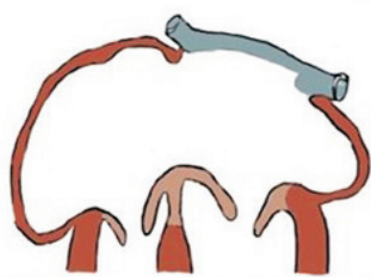

C Absence of SP and SS

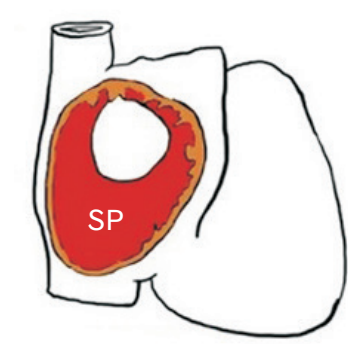

ASD

Fig. 2. Developmental defects of IAS formation showing. (A) Excessive resorption of SP. (B): Absence of SS. (C) Absence of both SP and SS, leading to a common atrium or Cor triloculare biventriculare. (D) Showing large FO. (E) Showing ASD. ASD, atrial septal defect; IAS, inter atrial septum; $\mathrm{O}$, foramen ovale; PV, pulmonary veins; RV, right ventricle; SP, septum primum; SS, septum secundum.

gradually disappears, the remaining part becomes the valve of the FO, which behaves like a shunt, which leads to oxygenated blood to flow from IVC directed through its eustachian valve to the LA passing through FO [2]. Soon after the birth, when the pressure in the LA increases, the two septa press against each other and this leads to closure of the communication between the two chambers [3]. Thus the rim of the fossa is prominent and often represents the edge of the fossa called septum secundum. It is most distinct above and in front of the fossa and usually deficient inferiorly. A small slit is sometimes found at the upper margin of the fossa, ascending beneath the rim to communicate with the LA. This represents the failure of obliteration of fetal FO, which remains patent in up to one-third of the normal hearts as patent foramen ovale (PFO) (Fig. 3) [4].

\section{Molecular regulation}

After bicuspid aortic valve, Atrial septal defects (ASDs) are the most common congenital heart disorder (CHD) [5]. Transcription factors regulating cardiac morphogenesis are NKX2.5, TBX5, and GATA4 which are important for understanding the etiology of human CHD. In families with ASD, numerous point mutations in NKX2.5 have been identified [6]. TBX5 gene mutations result in Holt-Oram syndrome, which is characterized by cardiac anomalies, similar to those with Nkx2.5 mutations (atrial and ventricular septal defects), 

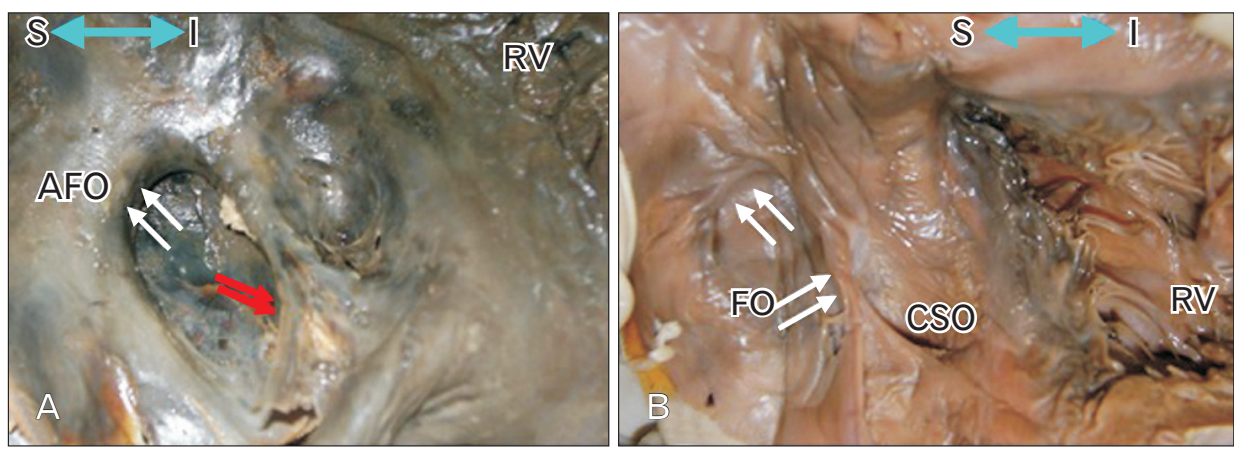

Fig. 3. Elliptical shape of FOv. (A) Showing raised margin in whole circumference AFO. In upper part shows slit like recess or pouch (white arrows). In lower part a deep recess present (red arrows). (B) Raised margin is present only in upper and anterior part of FO with slit like recess or pouch (white arrows). Blue double headed arrow showing S and I view. AFO, annulus fossa ovalis; CSO, coronary sinus opening; FO, foramen ovale; FOv, fossa ovale; RV, right ventricle; I, inferior; S, superior.

as well as preaxial limb abnormalities [7]. Mutations in the GATA4, a zinc-finger-containing protein, like the mutations of NKX2.5 and TBX5, cause similar atrial and ventricular septal defects in autosomal dominant non-syndromic human pedigrees [8]. FO occasionally may close prematurely during intrauterine life, which leads to massive hypertrophy of the RA and ventricle and underdevelopment of the left side of the heart. These babies usually do not survive [3].

\section{Clinical relevance}

In approximately $15 \%$ to $35 \%$ of individuals probe patency of FOv may be present. But few clinical studies say that FO is found to be present to a varying degree, which leads to mixing of oxygenated and deoxygenated blood, causing various clinical and subclinical conditions like migraine, cryptogenic stroke, decompression sickness in divers, and platypnea orthodeoxia [9]. In various studies, the presence of Patent PFO is a leading cause of paradoxical embolism and of cerebral emboli in the stroke of unknown origin and transitory ischemic attack $[10,11]$.

During raised pressure in the right heart (Valsalva maneuver) in diving, squatting, coughing, there is the direct transfer of venous blood from RA to LA through PFO, which leads to the neurological events associated with a paradoxical embolism [12]. Association has been reported between the size of PFO, the magnitude of the right-to-left shunt, and the presence of atrial septal aneurysm with increased chances of cerebral stroke [13]. Data suggest that in the neurological events associated with PFO, interventional treatment is better than the medical treatment, where the Percutaneous Transcatheter Closure technique of PFO is preferred [14]. For a correct trans-septal catheterization technique, echocar- diography is mandatory to localize the exact position of the fossa ovalis and to measure all other dimensions carefully before selecting the device.

Hence, morphometric analysis of FOv is potentially useful in guiding pediatric cardiac surgeons to correct ASD and as an aid for diagnosing prenatal narrowing or closure of FO [4]. The main aim of our study is to evaluate the morphology of FOv which will help the operating surgeons to know the exact location of FOv, its point of reference, and morphometric parameters to help them to assume precisely its dimensions for the selection of an appropriate device for closure of FOv defects.

\section{Materials and Methods}

Fifty apparently normal cadaveric hearts with age range of 50 to 80 years were examined, from the department of Anatomy over the period of 3 years, which were used for undergraduate teaching program and were preserved in $10 \%$ formalin. The RA was opened by taking at first a horizontal incision near the upper margin of the right auricle. The incision was further extended towards the right along the sulcus terminalis and inferiorly downs through the lateral edge of the RA up to its junction to IVC. The cut flap was reflected downwards towards the left to expose the interior of the RA. The cavity of the RA was washed thoroughly, and we made observations mentioned below:

1. Position of FOv concerning the right surface of the interatrial septum was noted i.e. middle of the interatrial wall, displaced towards the opening of IVC, displaced towards the opening of superior vena cava.

2. The shape of FOv was observed (circular, oval, irregu- 


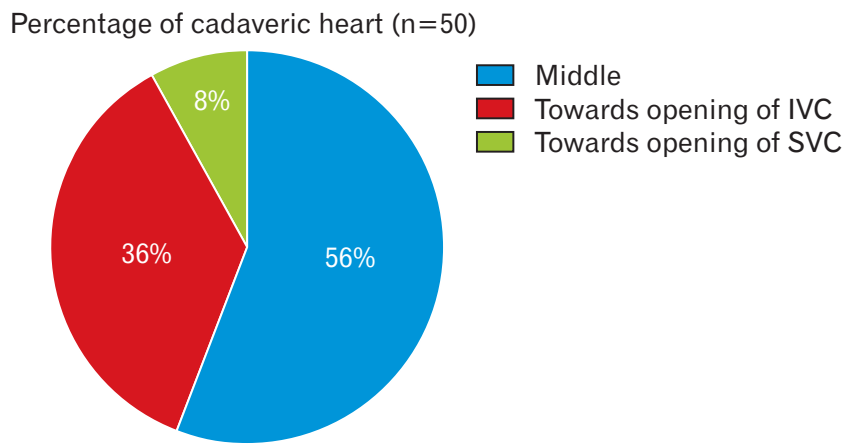

Fig. 4. Showing Position of FOv in the interatrial septum in cadaveric hearts $(n=50)$. FOv, fossa ovale; IVC, inferior vena cava; SVC, superior vena cava.

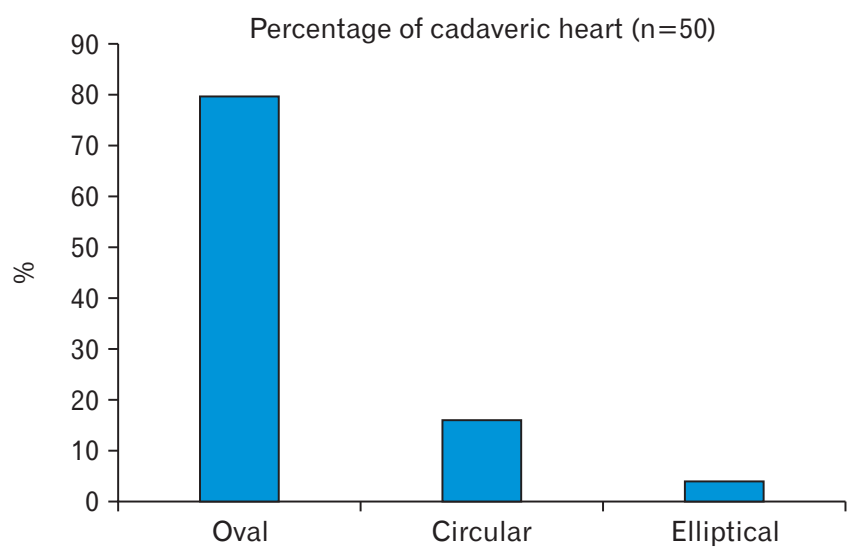

Fig. 5. Showing different shapes of FOv in the interatrial septum in cadaveric hearts $(n=50)$. FOv, fossa ovale.

lar, elliptical).

3. Size of FOv was measured (maximum length, maximum width) with the help of vernier caliper.

4. The extent, location, and thickness of limbus FOv were noted. The thickness of the limbus was measured with the help of the vernier caliper.

5. The thickness of the floor of FOv was measured with a vernier caliper and confirmed with a trans-illumination test where a torchlight was thrown into the FOv through the LA and transparency was confirmed. The floor was classified into three different types depending upon the thickness of the floor of FOv a) thick when more than $5 \mathrm{~mm}$, b) moderately thick between 2 to $4 \mathrm{~mm}$, and c) thin less than $1 \mathrm{~mm}$ with trans-illumination test positive.

6. Probe patency was confirmed by inserting a wire probe through the margins of FOv.

7. The redundancy of FOv was observed in each heart specimen.

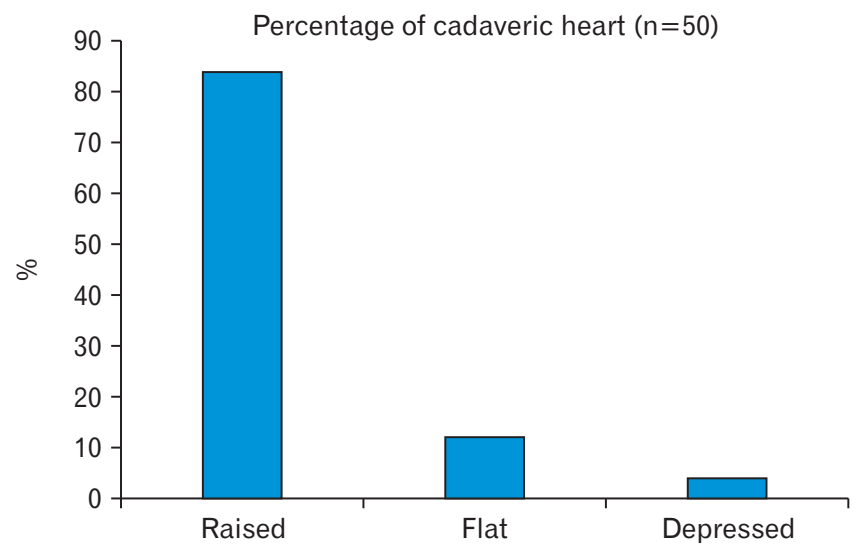

Fig. 6. Showing extent and location of Limbus fossa ovalis in the interatrial septum in cadaveric hearts $(n=50)$.

8. The associated structures in FOv like pouch, recess, fibrous bands, or membrane were also noted.

\section{Results}

Fifty human cadaveric heart specimens were carefully examined, with observations as below:

Position of fossa ovalis (FOv): In the present study the FOv occupied the middle of the interatrial wall in 28 (56.0\%), displaced towards the opening of IVC in 18 (36.0\%), and displaced towards the opening of superior vena cava in 4 heart specimens (8.0\%) (Fig. 4).

Shape of fossa ovalis: Shape was variable. It was oval in 40 $(80.0 \%)$, circular in $8(16.0 \%)$ and elliptical in 2 heart specimens (4.0\%) (Fig. 5).

Size of fossa ovalis: In the present study, the average transverse diameter of FOv was $24.21 \mathrm{~mm}$ (range, 12-26mm) and average vertical diameter was $26.84 \mathrm{~mm}$ (range, 14-28 mm).

Extent \& location of limbus: The limbus was found to be flat, raised or depressed at the margin. It was raised in 42 $(84.0 \%)$ and flat in $6(12.0 \%)$ and depressed in 2 specimens (4.0\%) (Fig. 6).

The prominence of the limbus: The limbus was prominent all around the margin in $7(14.0 \%)$, anterosuperior in 6 (12.0\%), anterior, superior, and inferior in 26 heart specimens (52.0\%) (Figs. 3, 7, 8).

The floor of Fossa ovalis: The floor was thick and muscular in 15 (30.0\%), moderately thick in 26 (52.0\%), and thin in 19 heart specimens (38.0\%).

PFO: FO was patent in 3 (6.0\%) (Fig. 10) whereas 12 specimens $(24.0 \%)$ had only probe patency. 

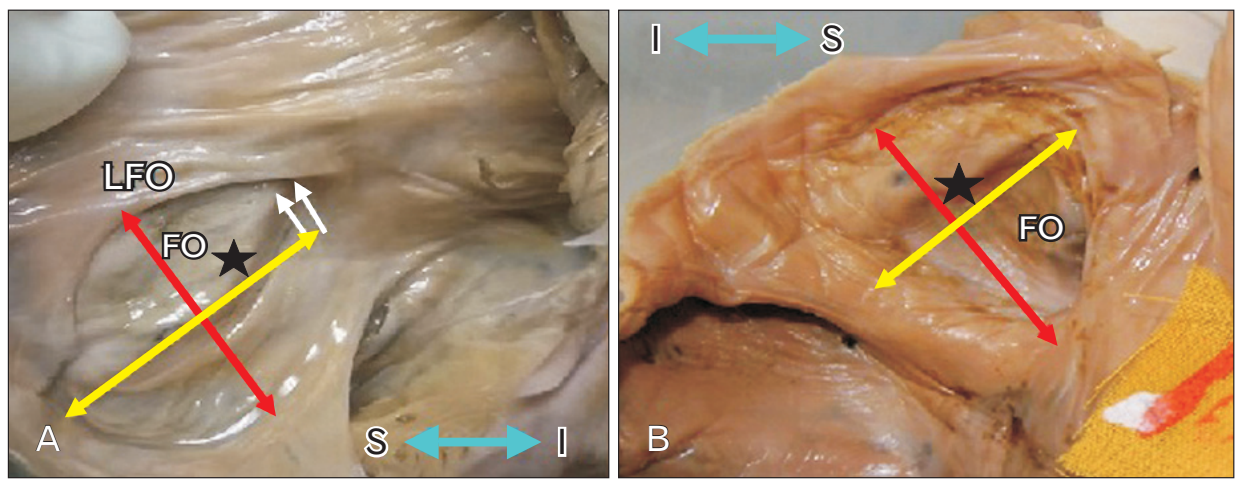

Fig. 7. Large FOv. (A) Showing deep recess in the superior margin (two white arrows), redundancy present in the middle part (asterisk). (B) Showing redundancy in the right part of FOv. In the right part showing bulging towards left atrium. Red arrow is showing vertical diameter, Yellow arrow is showing transverse diameter. Blue double headed arrow showing S and I view. FO, foramen ovale; FOv, fossa ovale; I, inferior; LFO, limbus fossa ovalis; S, superior.
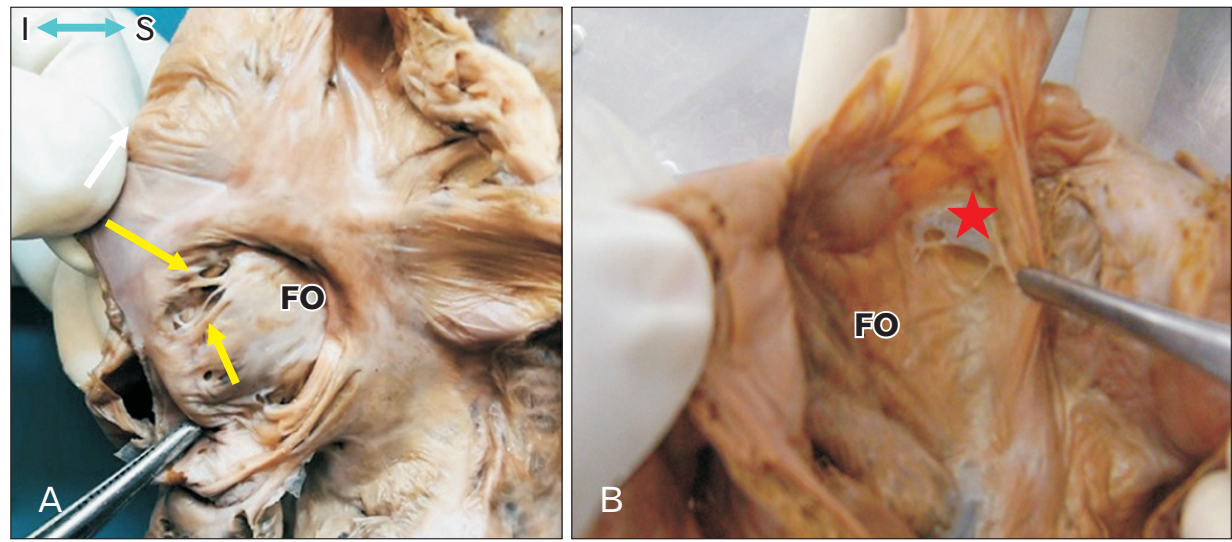

Fig. 8. (A) Showing fibrous brands in the inferior part (yellow arrows). (B) Showing a membrane (red asterisk) in antero superior part of FOv. Blue double headed arrow showing $S$ and I view. FO, foramen ovale; FOv, fossa ovale; I, inferior; S, superior.
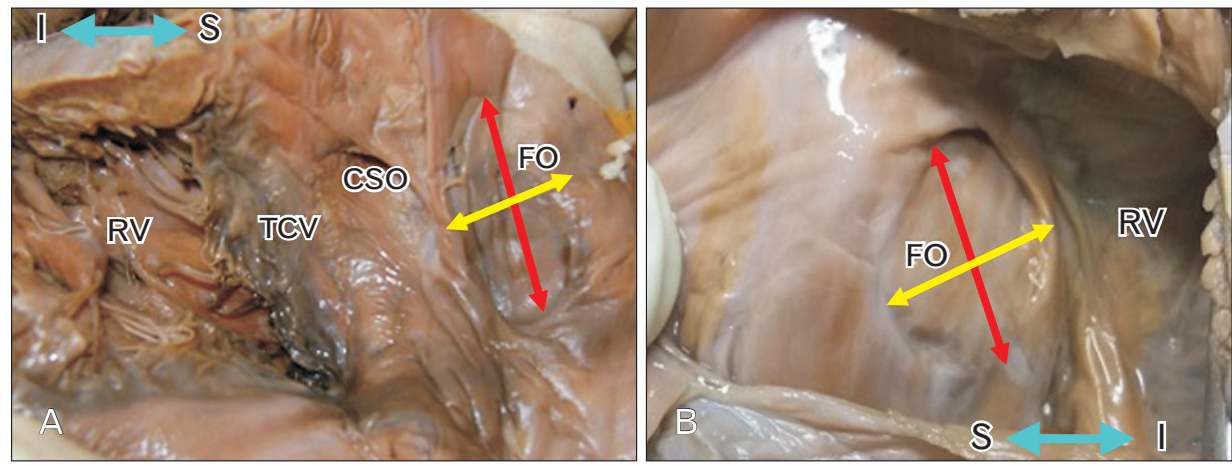

Fig. 9. Showing shapes of FOv. (A) Oval shape, (B) Round. Red arrow is showing vertical diameter, Yellow arrow is showing transverse diameter. Double-headed red arrow indicates the long axis and yellow arrow indicates width. Blue double headed arrow showing S and I view. CSO, coronary sinus opening; FO, foramen ovale; FOv, fossa ovale; I, inferior; RV, right ventricle; S, superior; TCV, tricuspid valve.

Recess/pouch: Concerning annulus fossa ovalis, deep recesses were observed (about $5-10 \mathrm{~mm}$ deep) in $8(16.0 \%)$ specimens, only like a slit along a part of annulus in $5(10.0 \%)$ heart specimens and in $3(6.0 \%)$ specimens pouch or pocket was present deep to annulus (Figs. 3, 7, 8, 9, 10).

Fibrous bands and membrane: FOv of 3 heart specimens 

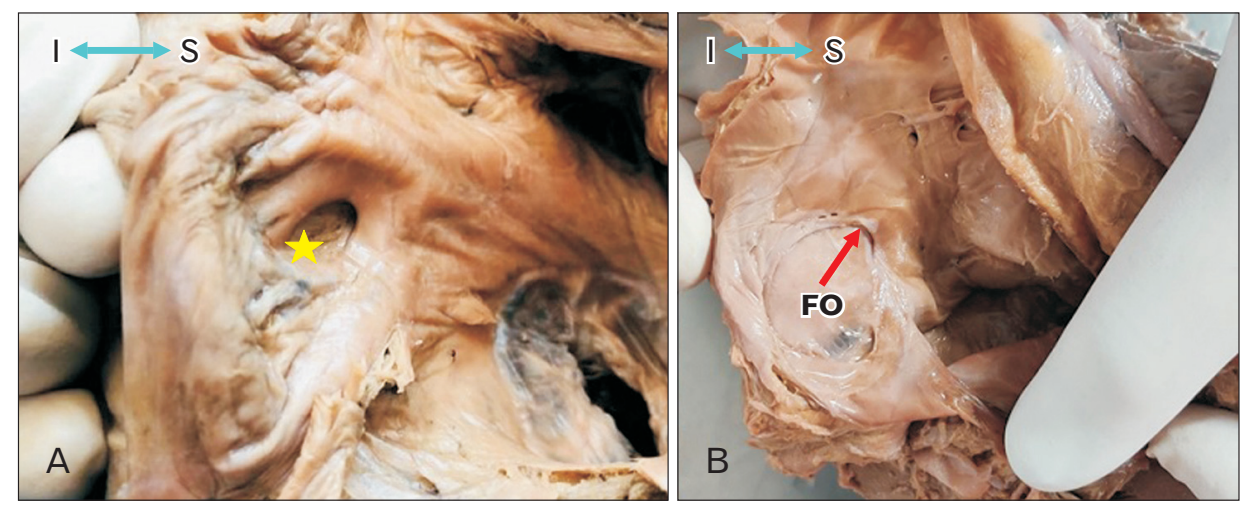

Fig. 10. Right surface of the interatrial septum. (A) Showing presence of a foramen (yellow asterik). (B) Showing deep recess (red arrow). Blue double headed arrow showing $S$ and I view. FO, foramen ovale; I, inferior; S, superior.

showed the presence of fibrous bands extending from posterosuperior and inferior part in one specimen approximately $1 \mathrm{~cm}$ in length, and in the second specimen was present in anterosuperior part (Fig. 8) where it formed a thin membrane also and in third specimen small bands seen along the posteroinferior and superior part of fossa ovalis (Fig. 10). These bands formed branching and rebranching patterns forming a fibrous network on the right surface of FOv.

Redundancy: The floor of the fossa was lax or folded in 5 (10.0\%) specimens (Fig. 7).

\section{Discussion}

Interatrial communications usually include a range of different atrial septum pathologies varying from PFO to true defects of interatrial septum within the FOv, the secundum ASDs, and defects of interatrial septum outside the region of FOv, such as the ostium primum defect and the sinus venosus defect (Fig. 2) [15]. The PFO, defined as an incomplete adherence of septum primum and septum secundum at the level of the fossa ovalis, is a common finding in the general population with a prevalence of about $25 \%$ [16]. PFO is the main cause of right to left cardiac shunts followed by pulmonary arteriovenous fistulas, and it is potentially a risk factor for paradoxical embolism. Classical clinical presentations of PFO include cryptogenic stroke, decompression syndrome, platypnea-orthodeoxia syndrome, and peripheral embolism. Recently, other associations like migraine with aura became a matter of investigation [17]. Isolated ASD represent $7 \%$ of all cardiac anomalies and can present at any age [15]

After the successful introduction of interatrial cardiac catheter techniques to treat CHDs, methods were evolved for the closure of ASDs percutaneously. For correct transseptal catheterization technique, it is essential to know the exact location of FOv, the point of reference, and those morphological parameters which allow us to assume precisely its dimensions [18].

Our study confirms the great variability in the morphology of FOv in cadaveric hearts. The FOv showed variability in its position concerning the interatrial septum. In most of the hearts, it was noted to be present in the middle part of the interatrial septum (56\%), followed by displacement towards the opening of IVC (36\%) and towards SVC (8\%), similar to the findings observed by Kanani et al. [19]. The FOv situated near the orifice of IVC will not only make surgeons difficult to distinguish the defect from an interatrial communication of inferior sinus type but such defect would also be difficult to close without impeding flow from the IVC. In these cases, the poster inferior rim may be too small to anchor the device satisfactorily without leaving a residual shunt. This was also true for the specimens in which the oval fossa was situated adjacent to the entrance of the SVC. Fraisse et al. [20] stated that the defects (ASDs) with deficient posteroinferior rim are not suitable for transcatheter closure as there is a considerable risk of device embolization. According to Chan and Goodman [21], ASDs displaced towards the superior and IVC veins affect the feasibility of transcatheter closure. Because the placement of the clam-shell device may obstruct these structures and recommended to have at least a $4 \mathrm{~mm}$ distance between the edges of the defect and SVC \& IVC [20].

In selected patients, interventional cardiology has made it possible to close ASDs with devices inserted through catheters for which anatomical knowledge of the types of defects in the FOv and surrounding structures is very much 
required. We observed variations in the shape of FOv as oval being most common in 40 (80.0\%), circular in 8 (16.0\%), and elliptical in 2 specimens (4.0\%) (Fig. 5), which were similar to findings of Joshi et al. [22]. The average transverse diameter of FOv observed in our study was $24.21 \mathrm{~mm}$ (range, 12-26 $\mathrm{mm}$ ) and the average vertical diameter was $26.84 \mathrm{~mm}$ (range, 14-28 mm). Whereas the dimensions observed by Kydd et al. [23] were anteroposterior $17 \mathrm{~mm}$ and supero-inferior $19.4 \mathrm{~mm}$ and other authors observed the average transverse diameter to be $14.53 \mathrm{~mm}$ and average vertical diameter to be $12.60 \mathrm{~mm}$ [22].

Limbus fossa ovalis was found to be raised in most of the heart specimens (84\%) in the present study, flat in $12 \%$, and depressed only in $4 \%$. The limbus was prominent all around the margin in $14 \%$, anterosuperior in $12 \%$, anterior, superior, and inferior in 26 heart specimens (52\%). In a study by Joshi et al. [22], the limbus was raised all-round in 92\% cases and flat in $4 \%$ cases. Naqvi et al describe the formation of limbus FOv in their article that during the 7 th week of development remodeling occurs in the atrial roof between the septum primum and the superior cava [24]. The septum secundum (secondary septum) forms as an infolding of the atrial wall. It folds inward to the right of the septum primum to form the anterosuperior, superior, and posterior margins of the muscular rim (limbus fossa ovalis) of the oval foramen. Thus, the infolded septum secundum is overlapped completely on the left atrial side by the thinner flap-like septum primum that becomes the floor of the oval-shaped depression (oval fossa) formed by the infolded rim on the right atrial side.

The thickness of the rim is due to the extracardiac tissue, usually adipose tissue contained in this fold. The thickness varies between individuals. The rim thickness is an important parameter analyzed before the device closure of PFO. The rim thickness not only helps to define the morphological phenotype of PFO but also decides whether the device will fit properly for the complete closure of PFO. If the surrounding rim is excessively bulky, the disc of the device will not be able to fit properly against the FOv. A smaller size or softer type of device is considered in such cases [25].

In the present study, the floor of FOv was thick and muscular in 15 , moderately thick in 26, and thin in 19 heart specimens. Kanani et al. [19] found that the floor of the FOvwas very thick and muscular in 19, moderately thick in 13, and thin in 08 in 40 heart specimens examined. Aneurysm was not observed in any of the heart specimens in our study. Kim et al. [26] while describing the development of inter- atrial septum mentioned that ridges of persisting posterior mesocardium protrude from each side of the orifice of the common pulmonary vein into the atrial cavity. The left ridge is transient whereas the right ridge, also known as the spina vestibuli (vestibular spine), grows bigger and contributes to cardiac septation. The spina vestibuli becomes muscularised with the incorporation of additional mesodermal tissue and is merging with the septum primum at the atrioventricular junction makes the septum thicker [26]. Thus, we can say that variations in the thickness of the floor of FOv are attributed to the varied incorporation of these components in the development of interatrial septum.

In our study, FO was patent in 3 specimens whereas 12 hearts had only probe patency. 'Probe patency' which is due to incomplete closure of FO post-birth is found in more than $25 \%$ of adult hearts, which is considered as a normal variant. During fetal life, the interatrial connection is open and normally closes within the first two years of life [27]. The persistence of this fetal communication between the two atria leads to PFO, which if persist in adulthood may lead to symptoms if the shunt is significant. By the fifth decade, $75 \%$ of patients with PFO experience symptoms of dyspnea on exertion and may have complications like arrhythmias, pulmonary hypertension, and right-sided heart failure from right ventricular volume overload [28].

In the present study, we observed fibrous bands in the fossa ovalis of 2 specimens and a thin membrane in one of these heart specimens. These bands formed branching and rebranching patterns forming a fibrous network on the right surface of FOv concerning limbus. Studies say that these fibrous networks may be formed due to incomplete overlap and atrophy of various embryological components of the interatrial septum. Similar fibrous bands were observed in one heart specimen in a study [22] and 7.4\% cases (out of 135 heart specimens) in another study on interatrial septum [29]. This network of fibrous bands may interfere during transseptal catheter insertion. May present as an obstacle due to increased thickness of the septum, hence these variations must be kept in mind while doing any intervention in this region to avoid any complications. We would like to mention here that these networks are not similar to Chiari's networks, which as the literature says is a mobile, net-like structure that originates from Thebesian or Eustachian valve and extends up to crista terminalis or interatrial septum [30]. Whereas the fibrous network we found is related to limbus fossa ovalis only. 
Deep recess and pouch have been observed in few heart specimens in our study, the significance of which can be derived from very few available literature review where pouch on the interatrial septa has been defined as a portion of the septum, formed in the absence of the PFO which gets invaginated towards other side and is due to incomplete fusion of the embryological components of the interatrial septum [31]. Embryological and anatomical knowledge of the recess and septal pouch in the interatrial septum is important during manipulating this region as it is a thin-walled structure, little force during catheter insertion may lead to its damage. Another most important relation to be kept in mind is the relation of the root of ascending aorta with the pouch, which may lead to critical complications or rupture or tear of aortic sinus while guiding catheter through this pouch unknowingly [29].

In conclusion, clinical anatomy plays a great role in understanding the morphology of the interatrial septum, FOv, and associated variations, especially for the cardiac surgeons dealing with this region. When transseptal access is required to enter the LA from the RA, anatomical knowledge becomes essential for safe and efficacious intervention and will be useful for device selection in treating ASD and PFO. Our study depicts the morphological variations of FOv which would help the clinicians in a deeper understanding of the region as very few cadaveric studies are available in the literature at present. The morphometric data reported here provide the surgeons and echocardiographers with a greater margin of safety in the management of ASDs especially in surgical intervention on FOv.

\section{ORCID}

Prajakta Kishve: https://orcid.org/0000-0002-3873-3192

Rohini Motwani: https://orcid.org/0000-0002-2002-5198

\section{Author Contributions}

Conceptualization: PK. Data acquisition: PK, RM. Data analysis or interpretation: PK, RM. Drafting of the manuscript: PK, RM. Critical revision of the manuscript: RM. Approval of the final version of the manuscript: all authors.

\section{Conflicts of Interest}

No potential conflict of interest relevant to this article was reported.

\section{Acknowledgements}

We would like to thank our undergraduate student Pawan Kalyan Reddy for helping us in drawing digital diagrams of the development of the interatrial septum and its defects related.

\section{References}

1. Rosse C, Gaddum-Rosse P. Hollinshead's textbook of anatomy. 5th ed. Philadelphia: Lippincott-Raven; 1997. p. 473.

2. Moore KL, Persaud TVN. The cardiovascular system. In: Moore KL, Persaud TVN, editors. The Developing Human: Clinically Oriented Embryology. 7th ed. Philadelphia: Saunders; 2003. p.340-5.

3. Sadler TW. Cardiovascular system. In: Sadler TW, editor. Langman's Medical Embryology. 12th ed. Philadelphia: Lippincott Williams \& Wilkins; 2012. p.162-200.

4. Standring S, Gray H. Gray's anatomy: the anatomical basis of clinical practice. 40th ed. Edinburgh: Churchill Livingstone/ Elsevier; 2008. p. 964.

5. Nashat H, Montanaro C, Li W, Kempny A, Wort SJ, Dimopoulos K, Gatzoulis MA, Babu-Narayan SV. Atrial septal defects and pulmonary arterial hypertension. J Thorac Dis 2018;10(Suppl 24):S2953-65.

6. Schott JJ, Benson DW, Basson CT, Pease W, Silberbach GM, Moak JP, Maron BJ, Seidman CE, Seidman JG. Congenital heart disease caused by mutations in the transcription factor NKX2-5. Science 1998;281:108-11.

7. Mori AD, Bruneau BG. TBX5 mutations and congenital heart disease: Holt-Oram syndrome revealed. Curr Opin Cardiol 2004;19:211-5.

8. Garg V, Kathiriya IS, Barnes R, Schluterman MK, King IN, Butler CA, Rothrock CR, Eapen RS, Hirayama-Yamada K, Joo K, Matsuoka R, Cohen JC, Srivastava D. GATA4 mutations cause human congenital heart defects and reveal an interaction with TBX5. Nature 2003;424:443-7.

9. Davison P, Clift PF, Steeds RP. The role of echocardiography in diagnosis, monitoring closure and post-procedural assessment of patent foramen ovale. Eur J Echocardiogr 2010;11:i27-34.

10. Mirzaali M, Dooley M, Wynne D, Cooter N, Lee L, Haworth P, Saha R, Gainsborough N, Hildick-Smith D. Patent foramen ovale closure following cryptogenic stroke or transient ischaemic attack: long-term follow-up of 301 cases. Catheter Cardiovasc Interv 2015;86:1078-84.

11. Nighoghossian N, Perinetti M, Barthelet M, Adeleine P, Trouillas P. Potential cardioembolic sources of stroke in patients less than 60 years of age. Eur Heart J 1996;17:590-4.

12. Milev I, Zafirovska P, Zimbakov Z, Idrizi S, Ampova-Sokolov 
V, Gorgieva E, Ilievska L, Tosheski G, Hristov N, GeorgievskaIsmail L, Anguseva T, Mitrev Z. Transcatheter closure of patent foramen ovale: a single center experience. Open Access Maced J Med Sci 2016;4:613-8.

13. Overell JR, Bone I, Lees KR. Interatrial septal abnormalities and stroke: a meta-analysis of case-control studies. Neurology 2000;55:1172-9.

14. Nietlispach F, Meier B. Percutaneous closure of patent foramen ovale: safe and effective but underutilized. Expert Rev Cardiovasc Ther 2015;13:121-3.

15. Rigatelli G, Magro B, Oliva L. Anatomo-functional characterization of interatrial septum for catheter-based interventions. Am J Cardiovasc Dis 2011;1:227-35.

16. Marelli AJ, Mackie AS, Ionescu-Ittu R, Rahme E, Pilote L. Congenital heart disease in the general population: changing prevalence and age distribution. Circulation 2007;115:163-72.

17. Rigatelli G, Rigatelli G. Congenital heart diseases in aged patients: clinical features, diagnosis, and therapeutic indications based on the analysis of a twenty five-year Medline search. Cardiol Rev 2005;13:293-6.

18. Reig J, Mirapeix R, Jornet A, Petit M. Morphologic characteristics of the fossa ovalis as an anatomic basis for transseptal catheterization. Surg Radiol Anat 1997;19:279-82.

19. Kanani SD, Tank K, Patil DS, Nirvan AB, Dave RV. Cadaveric study of fossa ovalis. BJKines-NJBAS 2014;6:1-4.

20. Fraisse A, Latchman M, Sharma SR, Bayburt S, Amedro P, di Salvo G, Baruteau AE. Atrial septal defect closure: indications and contra-indications. J Thorac Dis 2018;10(Suppl 24):S287481.

21. Chan KC, Godman MJ. Morphological variations of fossa ovalis atrial septal defects (secundum): feasibility for transcutaneous closure with the clam-shell device. Br Heart J 1993;69:52-5.

22. Joshi SD, Chawre HK, Joshi SS. Morphological study of fossa ovalis and its clinical relevance. Indian Heart J 2016;68:147-52.

23. Kydd AC, Das P, Hoole S, Shapiro LM, Rana BS. Defining patent foramen ovale morphology using three-dimensional transoesophageal echocardiography and relationship to shunt size. Heart 2013;99(Suppl S2):A72.

24. Naqvi N, McCarthy KP, Ho SY. Anatomy of the atrial septum and interatrial communications. J Thorac Dis 2018;10(Suppl 24):S2837-47.

25. Rana BS, Shapiro LM, McCarthy KP, Ho SY. Three-dimensional imaging of the atrial septum and patent foramen ovale anatomy: defining the morphological phenotypes of patent foramen ovale. Eur J Echocardiogr 2010;11:i19-25.

26. Kim JS, Virágh S, Moorman AF, Anderson RH, Lamers WH. Development of the myocardium of the atrioventricular canal and the vestibular spine in the human heart. Circ Res 2001;88:395-402.

27. Anderson RH, Brown NA, Webb S. Development and structure of the atrial septum. Heart 2002;88:104-10.

28. Teo KS, Disney PJ, Dundon BK, Worthley MI, Brown MA, Sanders P, Worthley SG. Assessment of atrial septal defects in adults comparing cardiovascular magnetic resonance with transoesophageal echocardiography. J Cardiovasc Magn Reson 2010;12:44.

29. Klimek-Piotrowska W, Hołda MK, Koziej M, Piątek K, Hołda J. Anatomy of the true interatrial septum for transseptal access to the left atrium. Ann Anat 2016;205:60-4.

30. Bhatnagar KP, Nettleton GS, Campbell FR, Wagner CE, Kuwabara N, Muresian H. Chiari anomalies in the human right atrium. Clin Anat 2006;19:510-6.

31. Krishnan SC, Salazar M. Septal pouch in the left atrium: a new anatomical entity with potential for embolic complications. JACC Cardiovasc Interv 2010;3:98-104. 